\title{
An evaluation on the operation parameters of trephine cutting cornea based on biomechanical response
}

PENG SU ( $\sim$ supeng@bistu.edu.cn )

Beijing Information Science and Technology University https://orcid.org/0000-0002-0005-5445

Shijing Deng

Capital Medical University

Wei Yao

University of Strathclyde

Jian Li

National research center for rehabilitation technical aids

Lei Tian

Capital Medical University

Yang Yang

Beihang University

Research article

Keywords: Cornea, Keratoplasty, Trephination, Operation parameter, Trephine Force

Posted Date: June 14th, 2019

DOI: https://doi.org/10.21203/rs.2.10198/v1

License: () (1) This work is licensed under a Creative Commons Attribution 4.0 International License.

Read Full License 


\section{Abstract}

Background: The aim of this paper is to propose laws of trephine operation in order to obtain better microsurgical effects for keratoplasty. Methods: Based on a handheld trephine manipulator and a trephine robot integrated with the microforce sensor, the manual and robotic experiments are made taking porcine corneas as the test subjects. The effect of trephine operation parameters on the results reflected by biomechanical response and photomicrograph is discussed, and the parameters include linear velocity, rotating angle and angular velocity. Results: Using probability density functions, the distributions of the manual operation parameters show some randomness, and there is large fluctuation in the trephine force during the experiments. The biomechanical response shows regular trends in the robotic experiments even under different parameters, and compared to manual trephination, the robot may perform the operation of trephine cutting cornea more stably and produce a uniform cutting margin. Conclusions: Under different operation parameters, the cutting force shows different trends, and the optimal initial parameters that result in better trephine effects can be obtained based on the trends. Based on this derived law, the operation parameters can be set in robotic trephination, and the surgeons can also be specially trained to achieve a better microsurgical result.

\section{Background}

Trephination, an elaborate ophthalmic microsurgery, is one of basic operations in keratoplasty. Keratoplasty can be divided into penetrating keratoplasty (PK) and deep anterior lamellar keratoplasty (DALK). PK is one of the most successful examples of tissue transplantation, and DALK is considered an alternative procedure to $\mathrm{PK}$ for corneal pathologies not affecting the endothelium and descemet membrane [1, 2]. Some scholars have discussed the biomechanics of transplanted corneas after keratoplasty, and the interaction in the recipient donor interface can influence healing response $[1,3]$. Some endothelial cells have been lost in the trephination process, especially at the cutting edge [4], and it affects the effect of the surgery. However, modifying the surgical technique and instrumentation may be beneficial to minimize this loss [5].

Nowadays, many surgeries of trephine cutting cornea are still manual operations in keratoplasty, which still strongly depend on the surgeons' experience and technique. Evaluating the operation may provide insight into the contribution of trephination methods to decrease the likelihood of complications arising. A few researchers are exploring advanced techniques to achieve good trephine results, such as femtosecond laser technology, robotics, and so on $[6,7]$. The use of femtosecond laser has some advantages, and it can prepare different configurations with a smooth cutting margin and a precise depth, but there are some drawbacks in clinical surgery, for example diseased cornea may affect laser energy, and the applications may be limited due to negative pressure suction in trephination. Robotics, the most similar method of manual operation, may overcome some shortcomings of manual operation, but the main application limitation is to ensure the reliability of micromanipulation in clinical microsurgery. Hu et al. [6] designed a robotic manipulator for trephine cutting cornea, and one of micro motors drove the trephine to punch the cornea linearly, and the other could realize the rotary motion based on the gear 
transmission. The mechanism is ingenious, but it did not consider the rotation angle can be adjusted.

Korff et al. [8] studied a semiautomatic trephination system, where a soft tissue preserving saw combined with automatic adaptation of the cutting depth to protect the dura mater and reduce the bone gap. Taylor $\mathrm{R}$ et al. [9] described a surgical robotic device integrated a unique sensor, and it may operate autonomously and evaluate the operation in real time by the state of tissues and disturbance information.

In these studies, effect of operation parameters on the biomechanical response will be discussed thoroughly in this microsurgical process of trephination. The biomechanical response may be described by the forces and the torques, and some factors can affect the response for example intraocular pressure (IOP) and velocity of trephine cutting cornea, etc. Besides, the trephine force and torque are complicated due to three-dimensional contact between the trephine and the cornea in the process of trephine cutting cornea, and the surgical operation involves two actions of linear punching and rotary cutting. The force $F$ can be divided into three components $F_{x} F_{y}$ and $F_{Z}$ and they are in the $\mathrm{X}$-axis and $\mathrm{Y}$-axis and Z-axis direction, respectively. And the torque $T$ also contains three components of $T_{x}, T_{y}$ and $T_{z}$ rotating about the $X$-axis and $Y$-axis and $Z$-axis, respectively $[10,11]$. Among them, the vertical force $F_{Z}$ which is perpendicular to the cutting plane reflects the reaction force and the punching force from cut tissue, and vertical torque $T_{z}$ is one of the significant parameters to describe biomechanical response which determines the cutting force $F_{c}$ in the process [11]. In the experiments, the trephine force can be simplified as two components of the vertical force $F_{z}$ and the cutting force $F_{c}[12]$.

Trephine operation is important for surgery effect in keratoplasty, which should attract more considerable attention. Firstly, this paper shows manual trephination by a series of experiments on porcine corneas. The operation law on manual experiments is obtained based on a probability density function, which describes three parameters of the linear velocity, the rotating angle, and the angular velocity. Analyzing the trephine force that can be used as a basis for evaluating the surgical effect, the data is calculated to obtain the expected values of the parameters. And by performing experiments whose initial parameters are closest to the expected values, the resulting trephine forces and photomicrographs are discussed to evaluate surgical effect of the surgeon. Then, the optimal parameters of trephination are obtained by discussing the variation of the cutting force under different initial conditions in robotic experiments, and the advantages of robotic trephination are also shown by the experiments.

\section{Methods}

In the experiments, manual and robotic trephination experiments are made using a disposable trephine with the diameter of $7.5 \mathrm{~mm}$, and the test subjects are fresh porcine eyeballs. Although there are some differences between the human cornea and the porcine cornea [13], the porcine cornea can be able to discuss the effect of trephine operation on biomechanics because they have enough similarities in the mechanical response. In order to reduce the errors in many experiments, the eyeballs are injected with 
normal saline to make the intraocular pressure value reach the normal value, i.e., $20.6 \mathrm{mmHg}$, measured by a dynamic contour tonometer [14].

\subsection{Manual Experiments}

A handheld trephine system comprises a handheld trephine manipulator integrated with the microforce sensor (i.e., NANO 17, ATI company [15]), a high-definition (HD) image acquisition system, and a dataprocessing system, as shown in Fig.1. Considering the significant randomness in manual experiments due to different surgeons with different surgical experiences and style, ten surgeons perform the experiments using the handheld manipulator, and carry out PK-trephination and DALK-trephination in 50 eyeballs each.

In the experiments, the trephine force data are measured by the sensor, which is an expression form of biomechanical response and can be used as a judgment basis for the result. The operation actions of the manual trephination are recorded by the high-definition camera, and the image data are collected by the data-processing system, and the effective marks of the image are extracted to match with the data of the forces. According to the data analysis, the operation parameters of the manual experiments are obtained including linear velocity, rotating angle, and angular velocity, and finally, the distributions and the expected values can be calculated based on probability theory.

\subsection{Robotic Experiments}

In many surgeries, the robots can assist the surgeons in undertaking some tasks, such as suturing and cutting, it is required that a smart control, a force-sensing of the contact between instrument and tissue, and an ability to predict the outcome of operations[6]. An experimental system for robotic trephination has been designed, which comprise a surgical robot, a trephine manipulator, a data-processing system, and a control system, as shown in Fig.2. The surgical robot which has been described in detail in a previous paper can adjust the position and posture based on the movements of three rotating joints [15]. The manipulator that can perform a trephine operation as the surgeon contains a linear punching motion and a rotary cutting motion, and the motions can be controlled independently based on the control system integrating the STM32 Microcontroller Unit. It is important for a successful experiment that the rotational cutting is a concentric motion, and in the manipulator, the bearing and the holder are used reasonably to ensure that the trephine can obtain a stable rotational movement from the motor.

Because the trephine depth is not discussed in this paper, which is a key aspect for DALK, the experimental system for robotic trephination performs three groups of PK-trephination experiments only. In each group, 50 experiments are carried out, and one of the three expected parameters obtained in the manual experiments is varied while the other two are kept constant. By mathematical analysis of the experimental results, this study tries to find the variations of trephine forces under different initial parameters.

\section{Results}


In probability theory, a probability density function (PDF), or density of a continuous random variable, is a function that describes the relative likelihood of this random variable taking on a given value. PDFs can give the expected value which is more comprehensive than the mean value because it can reflect the distribution of the data.

\subsection{Statistics and analysis of operation parameters on manual experiments}

There is the randomness attributed to surgeon's experience, technique and involuntary tremble in microsurgery, so the data of manual experiments are analyzed by building a PDF. The statistics of operational parameters of manual trephine surgery are shown in table 1, where the parameters calculated are the mean values in each experiment. Probability graphics of the parameters are shown in Fig. 3.

Firstly, linear velocity is related to the vertical force $F_{z}$ applied by the surgeon, which is the main factor that affects the operation time. In DALK-trephination, the surgeons need to determine the trephine depth precisely, so that they have to perform a more careful operation. So the velocity is much smaller than in PK-trephination, and the distribution of the parameters is more concentrated, as shown in figure (a). Secondly, there is a smaller rotating angle in PK-trephination, which is about 75 percent of the angle in DALK-trephination, as shown in figure (b). The performance reflects that the surgeon chooses a special treatment for each type of surgery. Finally, the angular velocity is related to the habits of the surgeon formed by long-term performing of the operation, and if there is no special training, it is difficult to change with technology. Although there is an obvious difference in the rotating angle, figure (c) shows that the angular velocities of the two types are close, including the expected value and the distribution.

In general, the trephine force is affected by all the three parameters. The operation parameters for PKtrephination have lower probability density, and the surgeon seem to perform a more casual operation for PK-trephination than for DALK-trephination.

\subsection{Statistics and analysis of trephine force}

The statistics of trephine force are shown in table 2, where the value of the vertical force $F_{z}$ is the maximum in each experiment, and the cutting force $F_{c}$ is the maximum of the peak to peak value, which is calculated by dividing the vertical torque $T_{z}$ by the length of the arm applying the force $/(/=37.5 \mathrm{~mm})$. The PDF of the trephine force is shown in Fig.4.

In PK-trephination, the vertical force $F_{z}$ is greater than the value in DALK-trephination, because the reaction force is larger, in large part caused by the extrusion of the eyeball, and the reaction force increases with trephine depth, as shown in figure (a). But in figure (b), the cutting force $F_{c}$ is smaller in the process of PK-trephine surgery, and it is not affected by the trephine depth. Overall, in agreement with Fig. 3 above, the same result arises that the distributions of the trephine force are more concentrated in DALK-trephination.

3.3 The variation of the trephine forceunder different operation parameters 
The randomness is inevitable in the manual microsurgery, so it is necessary to explore the impact of the operation parameters on the results of the surgery. Based on the series of robotic experimental data, the variations of trephine forces are discussed under operation parameters, including linear velocity, rotating angle, and angular velocity. The findings suggest that there are similar variations between the vertical force $F_{z}$ and the cutting force $F_{o}$ so the section will show the change of the force $F_{c}$ under different initial parameters because it reflects the law without interference from the reaction force [11], as shown in Fig.5.

Firstly, the rotating angle and the angular velocity are constants, and the variation is discussed under different linear velocities. The constants are set with reference to the expected parameters derived from the manual experiments, i.e., $[\theta, \omega]=[1.00,9.87]$. The force $F_{c}$ generally increases with linear velocity, and when the velocity is in the interval $A B$ (i.e., $75 \mu \mathrm{m} / \mathrm{s}>v>250 \mu \mathrm{m} / \mathrm{s}$ ), the increasing trend is most obvious, as shown in figure (a). Secondly, the variation of the force is discussed under different rotating angles, and the other two parameters are set to be constants derived from table 1 , i.e., $[v, \omega]=[34.52,9.87]$. As shown in figure (b), the force $F_{c}$ shows fluctuations, that means the force shows a clear upward tendency before point $A$, and then declining significantly to reach the trough point $B$, and after point $B$, the force slowly increases again. In the curve, there are two troughs, point $O$ and point $B$, but point $B$ should be the optimal parameter because the angle is small enough to avoid an unstable operation. Thirdly, the variation is discussed under different angular velocities, where the constants are set to $[v, \theta]=[34.52,1.00]$. The force $F_{c}$ in general decreases with angular velocity, and before point $A$, where the velocity is $15 \mathrm{rad} / \mathrm{min}$, the decreasing trend is at its most obvious, as shown in figure (c).

\subsection{Experimental result of trephine cutting cornea}

The result of each experiment can show the variation of the trephine force in real time rather than the average of many results, so an experiment is selected for the analysis of the results. The manual experiment and the robotic experiment will be analyzed respectively.

The experiments whose initial parameters are closest to the expected values are chosen, including a PKtrephination experiment and a DALK-trephination experiment. The results of the two experiments are shown in Fig.6.

Analyzing the manual experiments, two experiments are selected whose initial parameters are closest to the expected values in table 1, where the initial parameters of PK-trephination $[v, \theta, \omega]=[34.50,1.02,9.85]$, and the parameters experiment of DALK-trephination $[v, \theta, \omega]=[21.70,1.27,9.75]$, and the results of the experiments are shown in Fig. 6 . In figures (a) and (b), the dotted lines and the solid lines represent the results from PK-trephination and DALK-trephination respectively.

In figure (a), DALK-trephination is carried out, which starts at point A and stops at Point B, and the trephine depth is about 50 percent of the corneal thickness after 22.55 seconds. Vertical force $F_{z}$ can clearly show an increasing fluctuation caused by rotation and significant volatility, and the force reaches the maximum at point $\mathrm{C}$, at about $16.48 \times 10^{-2} \mathrm{~N}$. In the first period, the peak is small because the surgeon 
needs to adapt by using trial and error. Furthermore, there are larger fluctuations where the upward trend of the peaks is obvious and the troughs are close to zero because the vertical velocity is so small that the surgeons find it difficult to exert a sustained extrusion force. In the PK-trephination, the force also show an increasing fluctuation and uncertain volatility, but the maximum of $F_{z}$ is greater due to deeper depth, and the troughs can be kept in the process of experiment because the depth is not considered. In figure (b), the curve of the cutting force $F_{c}$ shows an approximate periodicity, with almost always some volatility in each period due to the instability of the operation. In figure (c), the photo of the corneal sample slice from form DALK- trephination is shown and describes a jagged margin which corresponds to the rotary cutting motion in figure (a) and (b), and the findings suggest that there is similar result in PK-trephination [12].

Based on the variation of the cutting force under different operation parameters, the optimal initial parameters of the robotic experiment are chosen as $[v, \theta, \omega]=[20.00,1.30,33.50]$, and the experimental results are shown in Fig.7.

In figures (a) and (b), the effective range of the experiment is from point A to point B. However, the linear velocity is so small that it leads to an increased surgery time (about 90 seconds), and the number of rotations is so many that the result shows concentrated curves because the angular velocity is fast enough but the rotating angle is relatively small. In figure (a), the vertical force shows a growing fluctuation, and the trephine always presses the cornea during the experiment. The force $F_{z}$ reached the maximum at point $C$ at about $14.92 \times 10^{-2} \mathrm{~N}$, and the punching force $F_{p}$ reaches a maximum $2.30 \times 10^{-2} \mathrm{~N}$. Compared with Fig.6 (b), figure (b) shows a change in the law leading to a much more stable behavior, and the force $F_{c}$ has obvious an increasing trend, with a value less than $2.40 \times 10^{-2} \mathrm{~N}$. In figure (c), there is a more regular cutting margin under the optimal initial parameters that means minor damage about the cornea, and it shows that the analysis about variations of the cutting force is meaningful for trephination. Under the same experimental conditions, figure (c) shows a relatively vertical trend for cutting margin under the optimal parameters.

\section{Discussions And Conclusions}

The manual and robotic experiments of trephine cutting cornea are performed on porcine cornea, and this paper tries to reveal some of the laws in the microsurgery based on the performance evaluation of trephine operations. Some scholars have paid close attention to corneal biomechanical changes after operation and discussed effect of the interface and the edge on healing time of wounds $[3,17]$. However, trephine forces should also attract more attention, and be used as the basis to judge the surgical effects and help surgeons perceive the process, use a reference force to make the surgery safer $[11,18]$.

Different operation parameters, including linear velocity, rotating angle, and angular velocity, etc., can lead to different trephine forces. Using a probability density function to describe the operation parameters on manual experiments of PK-trephination and DALK-trephination is discussed, and which shows the habits of the surgeon formed by long-term operations, and that these habits are difficult to change without 
special training. The studies show some randomness in the process, and there is a significant difference in the linear velocity and the rotating angle during the two types of trephination particularly. The trephine force of manual experiments, shown as the vertical force and the cutting force, is analyzed using the same probability theory. The trephine force is the most significant parameter to evaluate the surgical effect. The distribution of the trephine force is more concentrated in DALK-trephination due to the need for a careful operation. The results of manual trephination are shown by two experiments whose initial parameters are closest to the expected values of two types. In general, there is some uncertain volatility seen by large fluctuations in the curves of the trephine force, and the cutting margins shown in the photomicrographs are apparently destroyed due to the variation of the force generated by the instability of manual operation.

A surgical robot can assist an operator to achieve a highly skilled result, and a unique sensing and control approach is necessary to perform delicate surgery when using such devices [19]. This paper describes a robotic system that can carry out the trephine operation stably. A series of experiments are performed wherein the behavior of robotic trephination using the manipulator is analyzed. Under different operation parameters, the cutting force shows different trends, for example, it increases with linear velocity and decreases with angular velocity. It verifies the law's prediction that better trephine effects can be obtained under the optimal initial parameters. Based on the law, some reasonable parameters can be determined which can be used to guide the surgeons.

By comparing the results of manual trephination experiments and robotic ones, including the change laws of the trephine force and the photomicrographs, the effectiveness of the manipulator has been evaluated. The robotic trephination has shown some advantages that can generate a small and stable cutting force, and it can increase the similarity of the donor and the recipient, so the robot may be developed to produce a more accurate and uniform cutting margin which helps to the biomechanical properties of the cornea as a whole [5].

From the evaluation of robotic trephination, it can be found that the operation parameters affect the surgical effect, such as the recovery time and astigmatism [3]. It is important to determine the reasonable operation parameters in manual trephination, and it is necessary to train surgeons referencing to these parameters. Although the effect of operation parameters on the biomechanics of trephine cutting cornea has been discussed, it needs to be further identified that the law and the robot are applied in clinical surgery. And there are more factors should be considered, including test in clinical practice, and the control of trephine depth, the initial pose of the trephine. We believe that the research carried out here within, and further research in this area, will promote the progress of technology of trephine cutting cornea for keratoplasty including manual skill, assisted robot, and so on.

\section{Abbreviations}

PK: penetrating keratoplasty; and DALK: deep anterior lamellar keratoplasty; PDF: probability density function. 


\section{Declarations}

\section{Acknowledgements}

None

\section{Funding}

The author wishes to thank Young Elite Scientists Sponsorship Program by CAST (Grant No. 2016QNRC001), China Postdoctoral Science Foundation (Grant No. 2018M641290), and Training plan of Young Top-notch Talent by Beijing Municipal Education Commission (Grant No. CIT\&TCD201704063) that supported this work.

\section{Ethics approval and consent to participate}

As this is a study of operation analysis that does not involve human participants, human data or human tissue, no ethical approval and consent were required. The test subjects are in vitro porcine eyeballs, the study accords with the principle of ethics.

\section{Availability of data and material}

The datasets used and/or analyzed during the current study are available from the corresponding author on reasonable request.

\section{Competing interests}

The authors declare that they have no competing interests.

\section{Authors' contributions}

Participated in study design: SP, DSJ, and YY. Conduct of the study: SP, DSJ, and TL. Performed data analysis: SP, YW, and LJ. Wrote or contributed to the writing of the manuscript: SP, YW and YY. All authors have read and approved the final manuscript.

\section{Consent for publication}

Not applicable. 


\section{References}

[1] Jafarinasab MR, Feizi S Javadi MA, Hashemloo A. Graft biomechanical properties after penetrating keratoplasty versus deep anterior lamellar keratoplasty. Curr Eye Res. 2011; 36(5):417-421.

[2] Shimmura S, Tsubota K. Deep anterior lamellar keratoplasty.[J]. Curr Opin Ophthalmol, 2006, 17(4):349-355.

[3] Abdelkader A. Influence of different keratoplasty techniques on the biomechanical properties of the cornea. Acta Ophthalmol. 2013; 91(7):e567-e572.

[4] Bourne WM. Morphologic and functional evaluation of the endothelium of transplanted human corneas. Trans Am Ophthalmol Soc. 1983; 81.

[5] Moshirfar M, Meyer JJ, Kang PC. A comparison of three methods for trephining donor corneal buttons: endothelial cell loss and microscopic ultrastructural evaluation. Curr Eye Res. 2009; 34(11):939-944.

[6] Hu Y, Li D, Yang Y, Sun X. Integration of microsensor for microsurgery robot's end-effector. Front Mech Eng China. 2007; 2(2):205-209.

[7] Soong, H. K., Malta, J. B., 2009, “Femtosecond lasers in ophthalmology," Am. J. Ophthalmol., 147(2), pp.189-197.

[8] Korff, A, Follmann, A, Fürtjes, T, Habor, D, Kunze, SC, Schmieder, K, Radermacher, K. Concept and evaluation of a synergistic controlled robotic instrument for trepanation in neurosurgery. IEEE Trans on Rob and Autom. 2011; 47(10):6258 - 6263.

[9] Taylor, R, Du, X, Proops, D, Reid, A, Coulson, C, Brett, PN. A sensory-guided surgical micro-drill. Proc. IMechE, Part C: J. Mechanical Engineering Science. 2010; 224:1531-1537.

[10] Galiana, I, Bielza, M, Ferre, M. Estimation of normal and tangential manipulation forces by using contact force sensors. Lect Note Comput Sci. 2010; 6191, 65-72.

[11] Su P., Yang Y., Zhang L., Huang L., Biomechanical simulation of needle insertion into cornea based on distortion energy failure criterion. Acta Bioeng Biomech, 2016, 18(1):65-75.

[12] Su P., Deng S., Huang L., Song Y., Liu X., YangG Y., Analysis and evaluation of a robotic trephination in penetrating keratoplasty, J. Med. Devices, 2016, 10(2).

[13] Elsheikh A., Alhasso D., Rama P., Biomechanical properties of human and porcine corneas, Exp. Eye. Res., 2008, 86(5), 783-790.

[14] Lim T-C, Chattopadhyay S, Acharya UR. A survey and comparative studyon the instruments for glaucoma detection. Med. Eng. Phys., 2012;34:129-39. 
[15] Ferre M, Galiana I, Aracil R. Design of a lightweight, cost effective thimble-like sensor for haptic applications based on contact force sensors. Sensors. 2011; 11(12):11495-11509.

[16] Yang, Y., Xu, C., Deng, S., Xiao, J., 2012, Insertion force in manual and robotic corneal suturing. Int J Med Robotics Comput Assist Surg. 2012; 8(1), 25-33.

[17] Abdelkader A, Elewah el-SM, Kaufman HE . Confocal microscopy of corneal wound healing after deep lamellar keratoplasty in rabbits. Arch Ophthalmol. 2010; 128(1):75-80.

[18] Abolhassani N, Patel R, Moallem M. Needle insertion into soft tissue: A survey. Med Eeg Phys. 2007; 29(4), 413-431.

[19] Brett P, Du X, Zoka Assadi M, Proops D, Reid A, Coulson C. Mechatronic hand-held surgical robots. Mechatronics and Machine Vision in Practice, 2012 19th International Conference. 2012; 447 - 449.

\section{Figures}

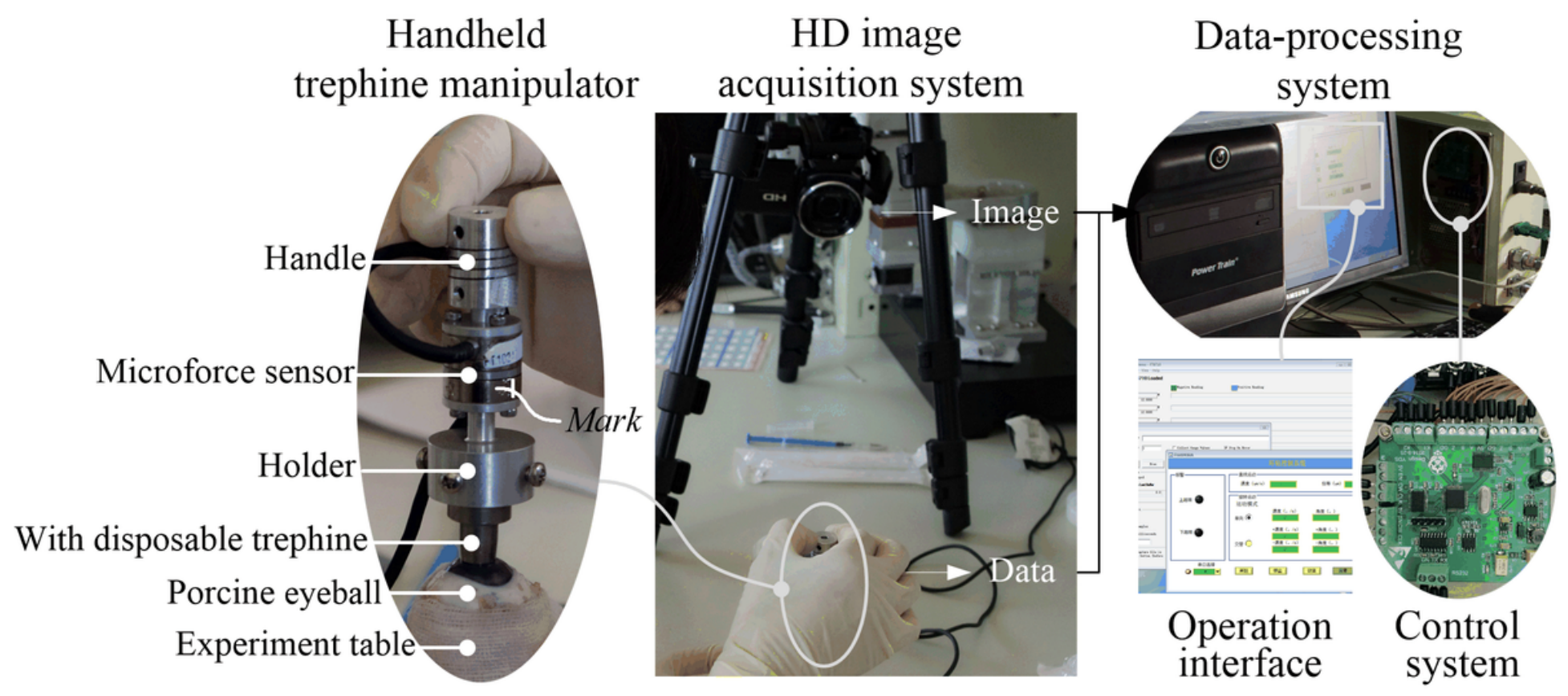

Figure 1

The experiment system of manual trephination 


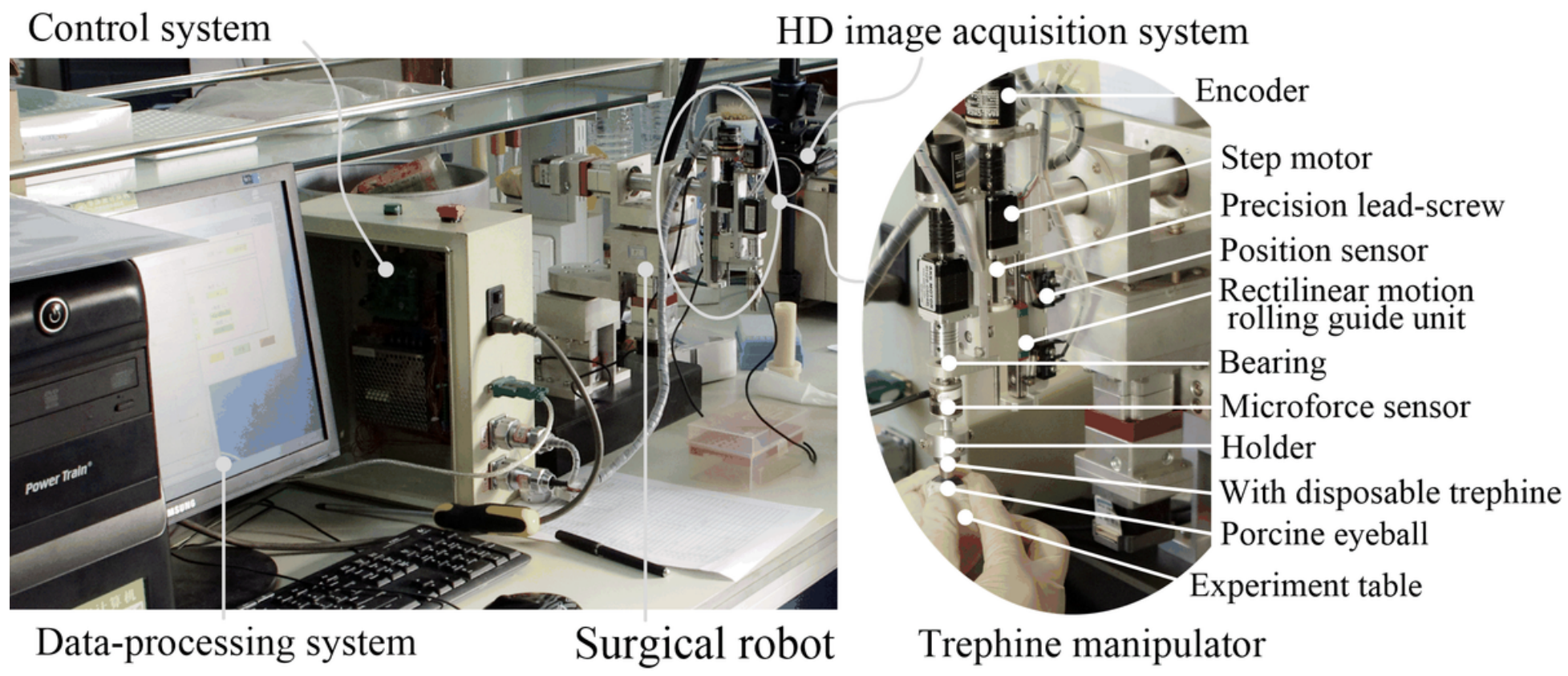

Figure 2

The experiment system of robotic trephination 


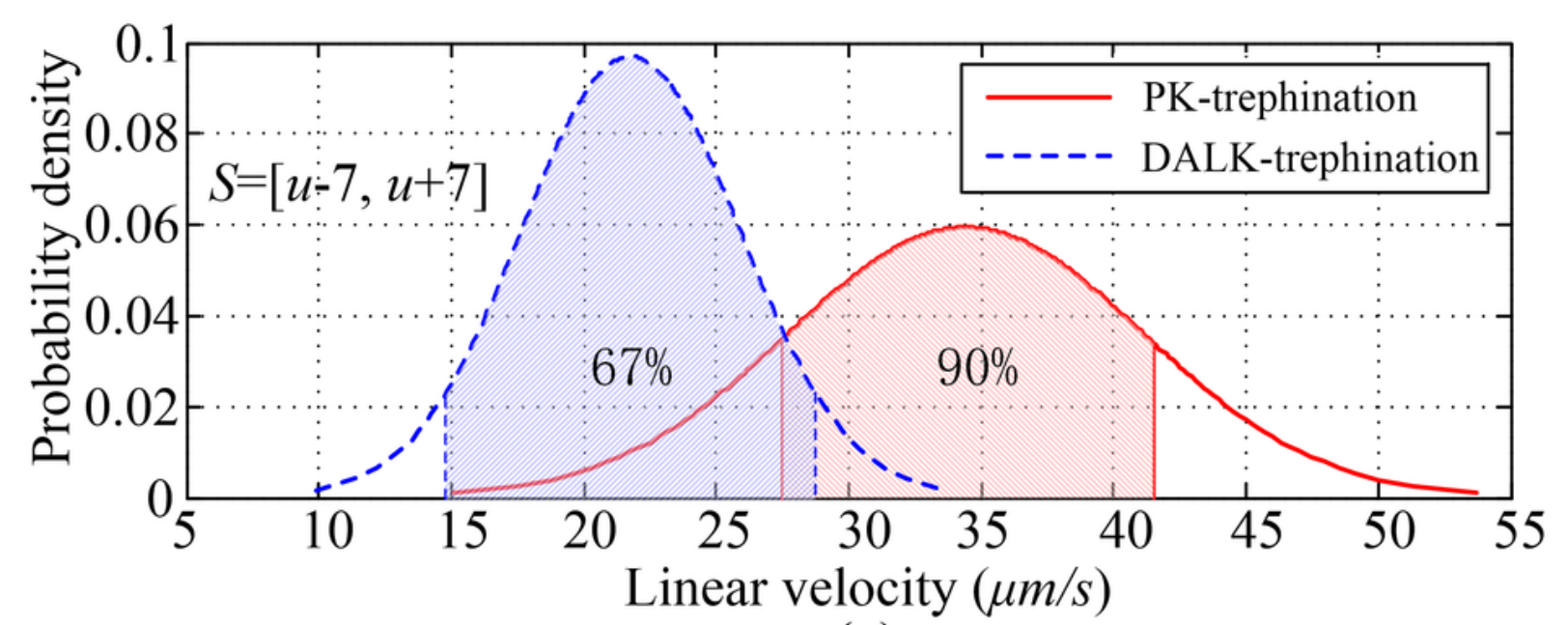

(a)

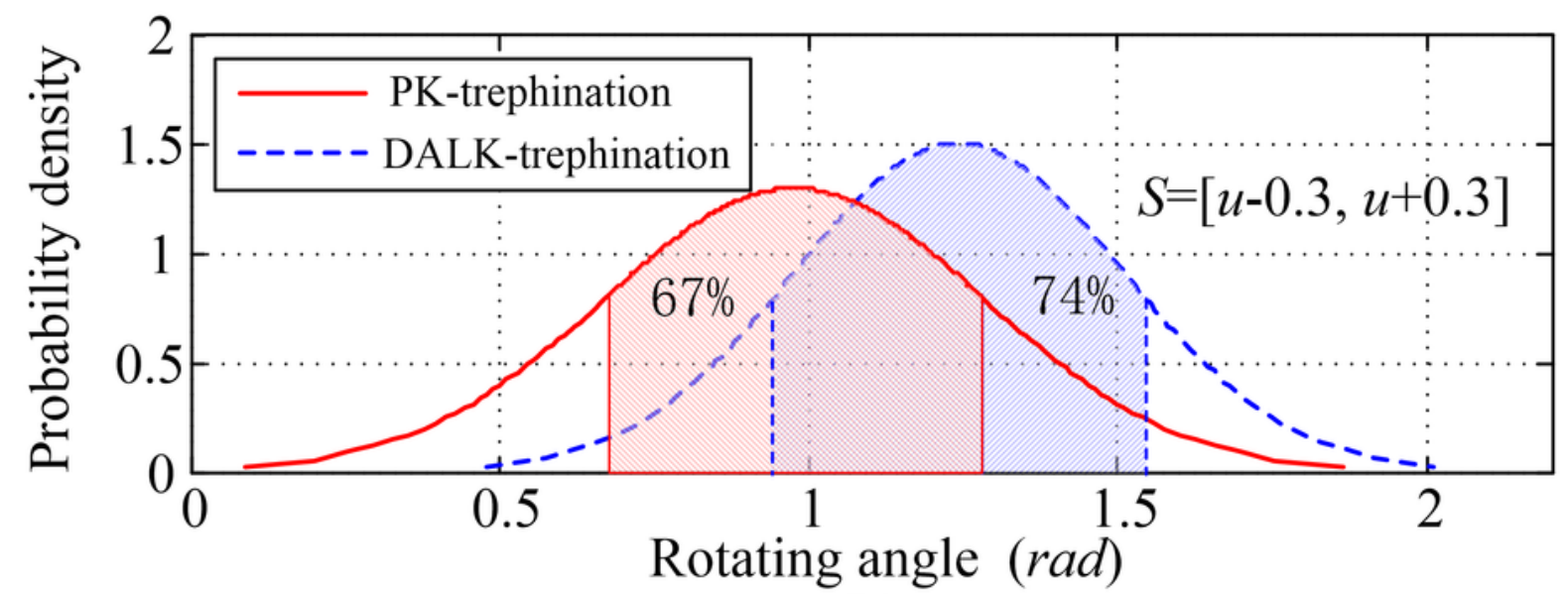

(b)

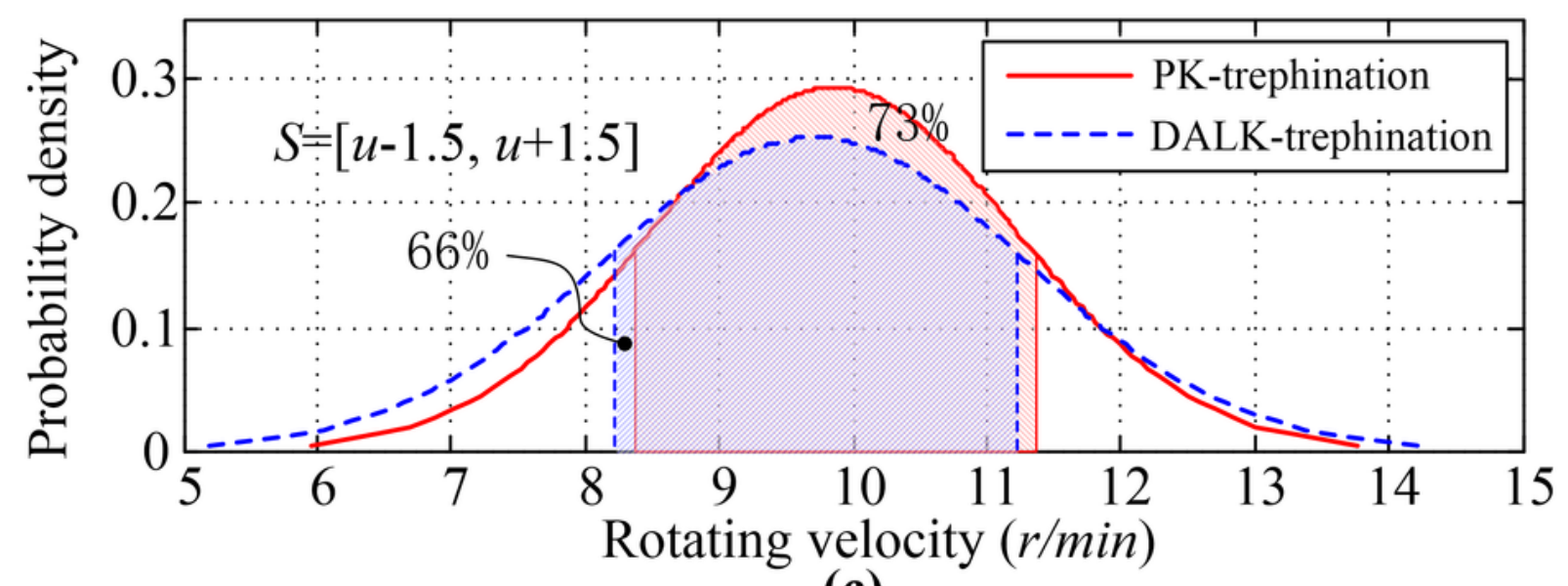

(c)

\section{Figure 3}

Probability graphics of operation parameters for manual experiments. The percentage, i.e., probability density $p$, is the probability value of a random variable being in the sample interval $S$. The figures (a), (b), and (c) describe the values of the probability density for the linear velocity, the rotating angle, and the angular velocity, respectively. 




(a)

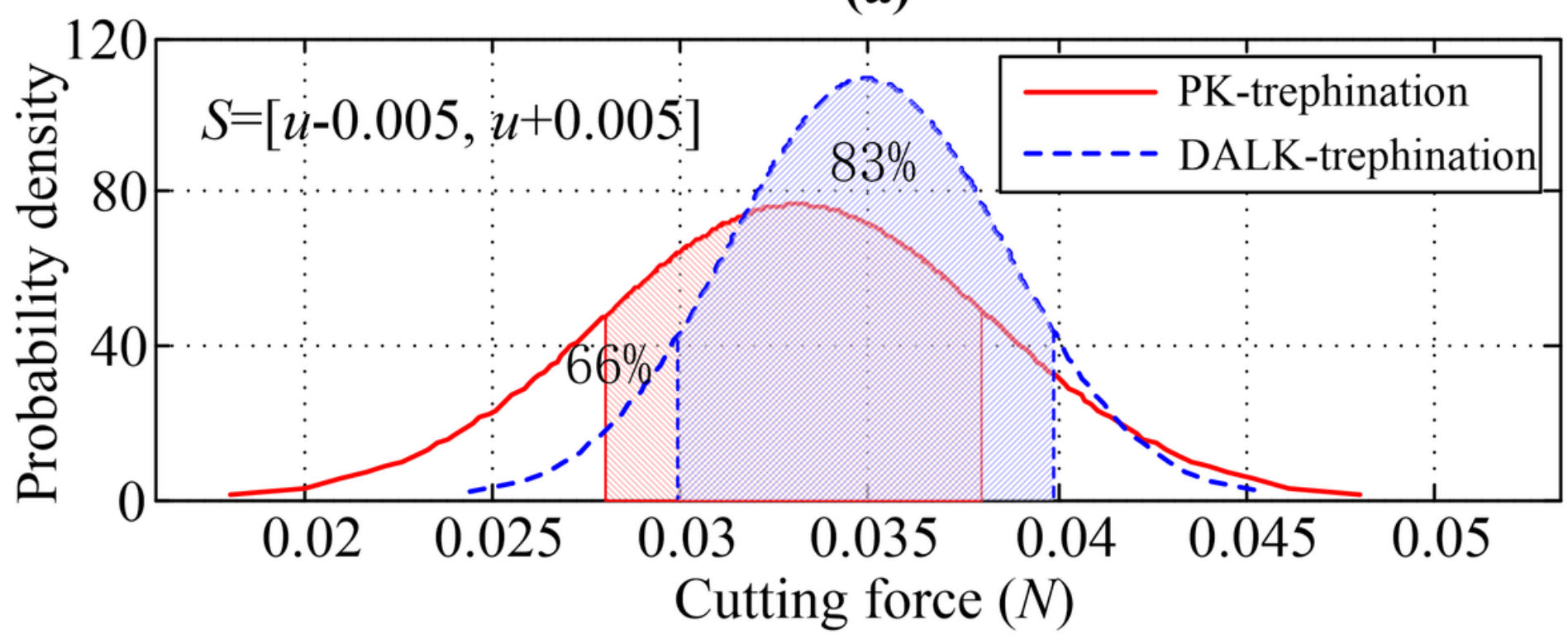

(b)

Figure 4

Probability graphics of the trephine force for manual experiments. The figure (a) and (b) describe the PDF for the vertical force Fz and the cutting force Fc, respectively. 

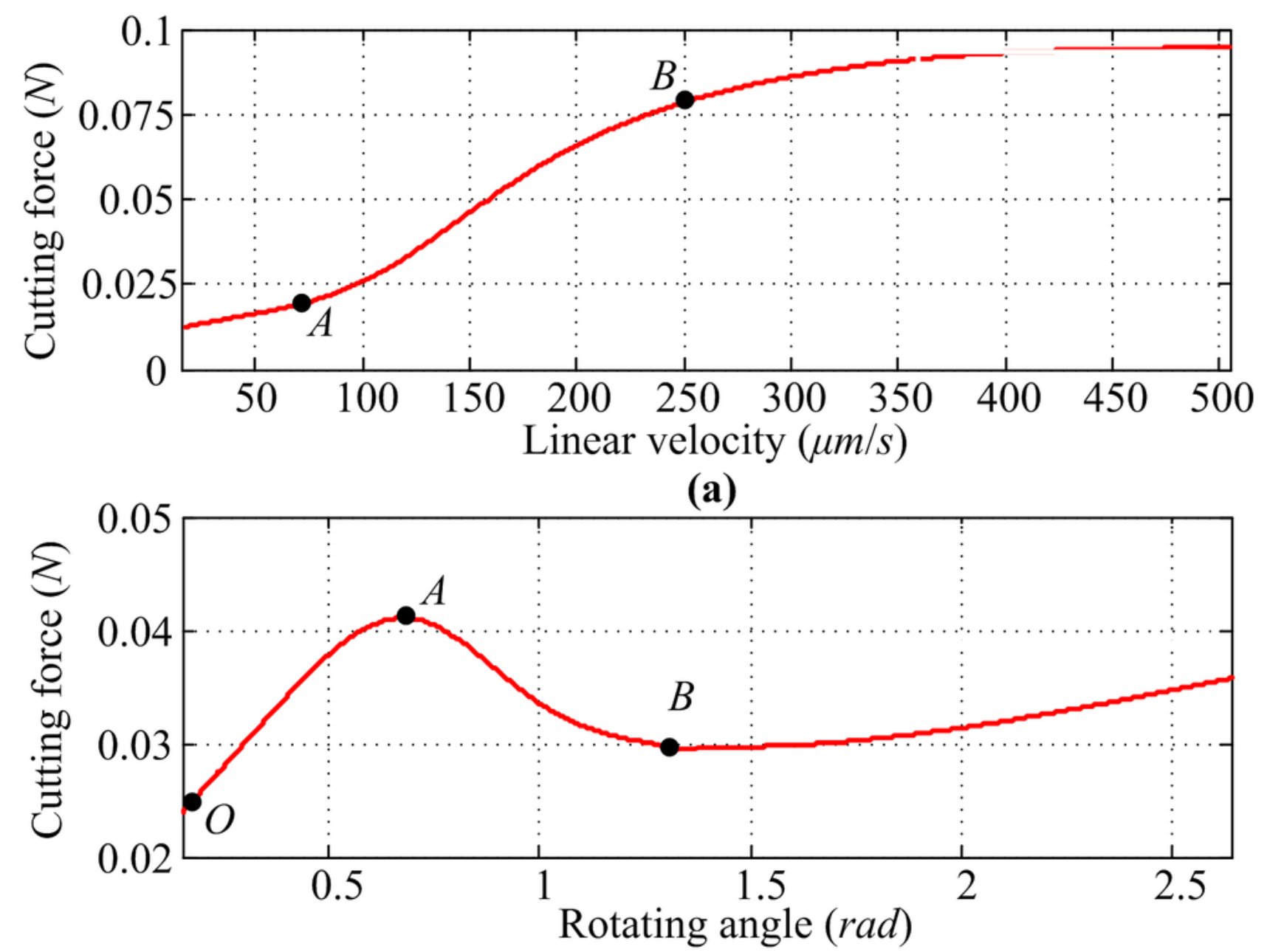

(b)



(c)

\section{Figure 5}

The variation of the cutting force Fc under different operation parameters. Figures (a), (b), and (c) are the results obtained by changing the linear velocity, the rotating angle, and the angular velocity, respectively. 


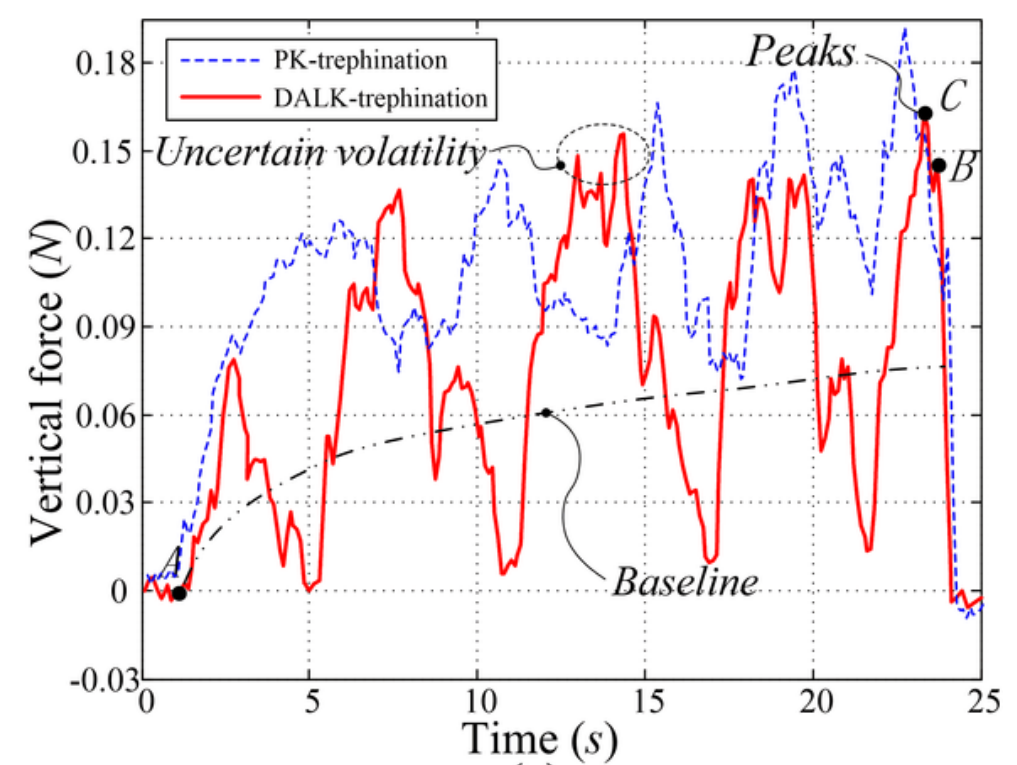

(a)



(b)

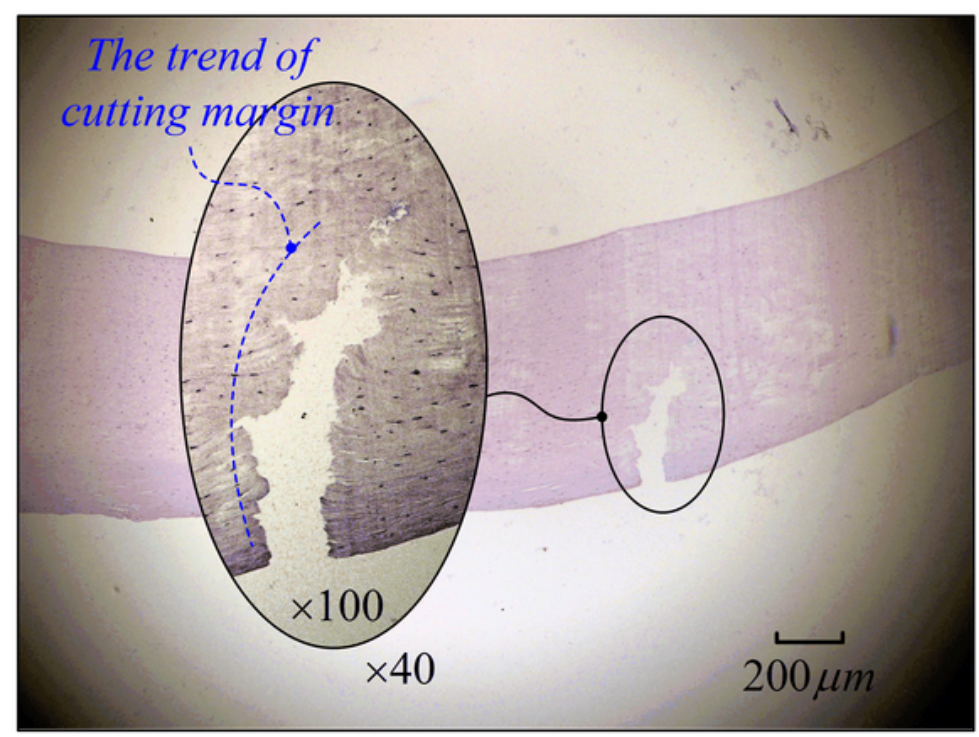

(c)

\section{Figure 6}

The results of the manual experiment under expected parameters. Figures (a) and (b) show the curves of vertical force Fz and cutting force Fc. Figures (c) is a photo of the corneal sample slice from DALKtrephination, which describes the photomicrographs obtained at an original magnification of $40 \times$ and $100 \times$. 


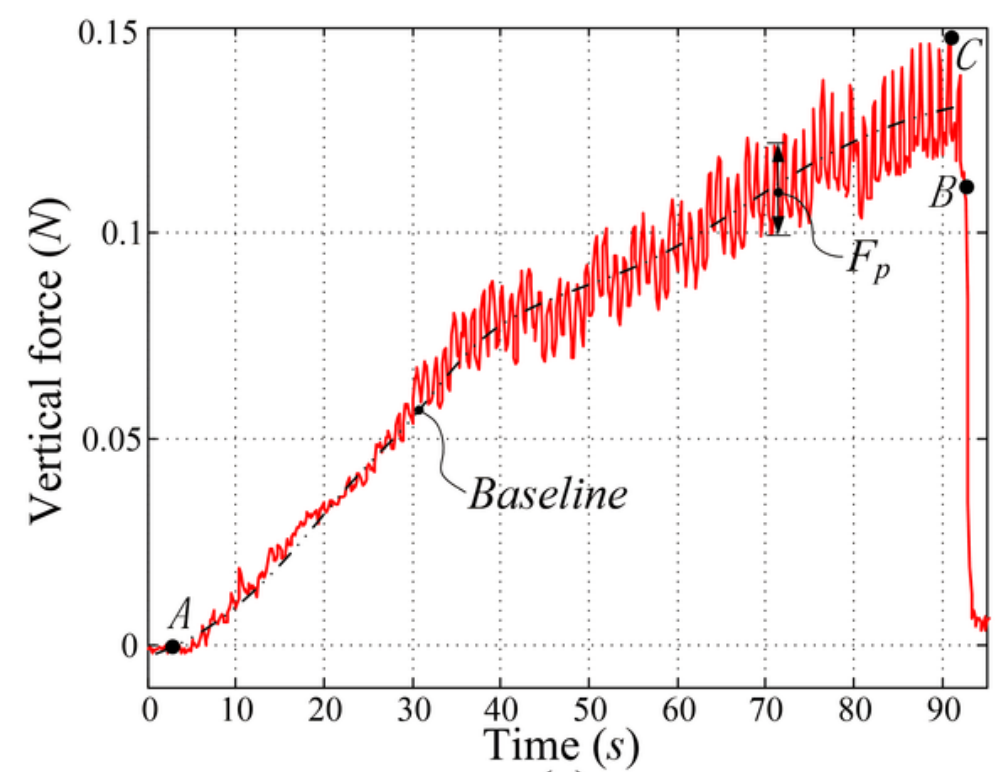

(a)



(b)

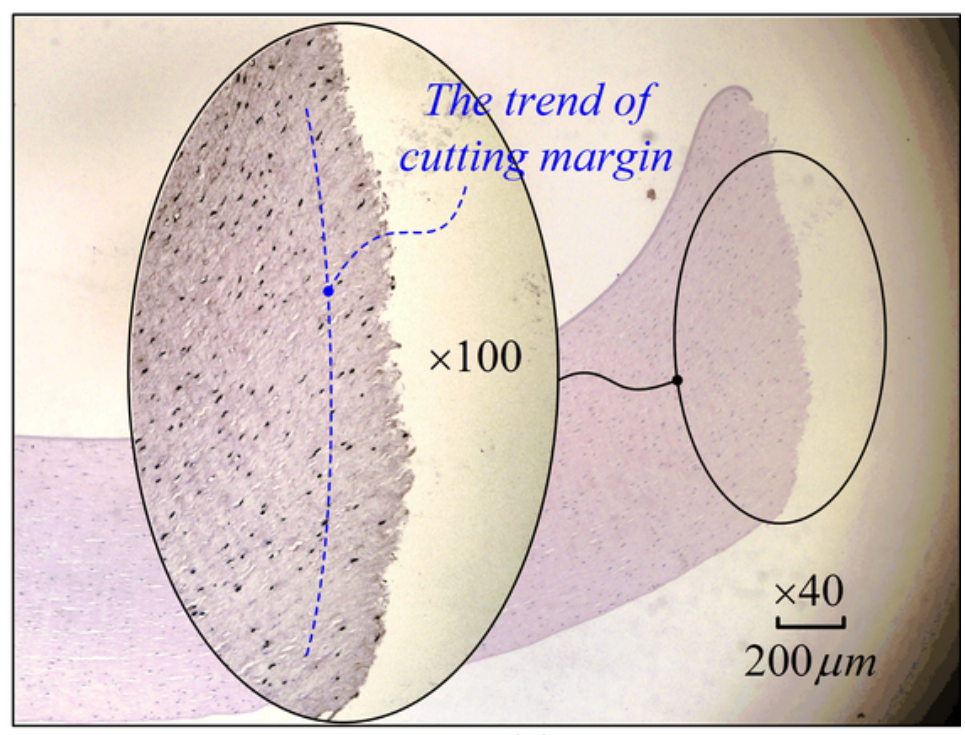

(c)

Figure 7

The results of robotic experiments under optimal initial parameters. Figures (a) and (b) show the curves of vertical force Fz and cutting force Fc. Figures (c) describes the photomicrographs obtained at an original magnification of $40 x$ and $100 x$. 